\title{
BIORREMEDIAÇÃO IN SITU COM PERÓXIDOS SÓLIDOS E SURFACTANTE DE SOLOS CONTAMINADOS
}

\author{
Tiago Bender Wermuth ${ }^{1}$ \\ Diosnel Antonio Rodriguez Lopet
}

\section{RESUMO}

O presente trabalho objetiva avaliar o uso de peróxidos sólidos com a adição de sais de fosfato, nitrogênio e surfactante na biorremediação de solos contaminados por derivados de petróleo. Para isso, coletou-se uma amostra do solo contaminado durante os trabalhos de recuperação de uma área de um posto de gasolina. A mesma foi colocada em uma caixa de PVC, dividida em duas partes. Uma parte foi tratada por meio da adição de peróxido de cálcio, surfactante e superfosfato (fonte de nutrientes). Já a outra recebeu a aplicação de somente peróxido de cálcio e superfosfato. Os resultados do tratamento deste solo foram monitorados semanalmente por meio de medidas da umidade, $\mathrm{pH}$ e temperatura. A descontaminação do solo foi acompanhada pela análise dos compostos Benzeno, Tolueno, Etilbenzeno, Xileno, TPH-GRO (Gasoline Range Organics), TPH-ORO (Oil Range Organics) e TPH-DRO (Diesel Range Organics), segundo as normas USEPA 8015D, USEPA 5021A, USEPA 8021B. Os valores mostram que o solo tratado com peróxido de cálcio, surfactante e superfosfato tiveram resultados semelhantes ao lado em que não continha o surfactante.

Palavras-chave: Biorremediação. Peróxido de Cálcio. Surfactante. Superfosfato.

\section{ABSTRACT}

The present work aims to evaluate the use of solid calcium peroxides along with addiction of phosphate salts, nitrogen and surfactant on bioremediation of soils contaminated by petroleum derivates. It was collected a sample of contaminated soil, during the recovery work of a gas station area. It was located in a PVC box, divided in two parts. One part was treated with the addiction of calcium peroxide, surfactant and superphosphate (source of nutrients). The other received the application of calcium peroxide and superphosphate only. The results of the treatment of this soil were weekly monitored, through the humidity parameters values, the $\mathrm{pH}$ and

1 Graduando em Engenharia Ambiental - UNISC, Bolsista PROBIC- FAPERGS; e-mail: tiago.haine@gmail.com.

2 Doutor em Engenharia de Materiais, Metalurgia e Meio Ambiente-Berlim-Alemanha, Professor do Departamento de Engenharia, Arquitetura e Ciências Agrárias e do PPGTA, UNISC; e-mail: dlopez@unisc.br. 
temperature follow up. The analysis of the Benzene, Toluene, Ethylbenzene, Xylene,TPH-GRO ( Gasoline Range Organics), TPH-ORO ( Oil Range Organics) and TPH-DRO ( Diesel Range Organics) compounds were performed at the Analytical Solutions Laboratory of the Veritas Bureau Group of São Paulo, SP, Br, according to the USEPA 8015D, USEPA 5021A, USEPA 8021B rules. The values shown that the soil treated with calcium peroxide, surfactant and superphosphate had similar reductions to the side without surfactant.

Keywords: Bioremediation. Calcium Peroxide. Surfactant. Superphosphate.

\section{INTRODUÇÃO}

Os crescentes problemas de contaminação de solo por hidrocarbonetos em postos de distribuição de combustíveis vêm sendo alvo de inúmeras pesquisas. Esse tipo de contaminação é muito preocupante uma vez que esses hidrocarbonetos podem se espalhar por grandes extensões, carregados pelas águas subterrâneas, comprometendo a qualidade desse recurso e a do solo. A aplicação de técnicas de biorremediação vem se destacando como uma das estratégias mais promissoras e eficazes ao tratamento de solos contaminados por hidrocarbonetos de petróleo.

Nesse sentido, o presente trabalho objetiva avaliar o uso de peróxidos sólidos de cálcio, juntamente com a adição de sais de fosfato, nitrogênio e surfactante, na biorremediação de solos contaminados por derivados de petróleo.

\section{FUNDAMENTAÇÃO TEÓRICA}

A contaminação de solos por hidrocarbonetos derivados de petróleo vem se constituindo um desafio para profissionais que atuam na área de saneamento ambiental, uma vez que os fenômenos geoquímicos e bioquímicos são complexos e aqueles são catalisados a partir de sua inserção no subsolo.

Quando os combustíveis atingem o solo, seus componentes separamse em três fases: dissolvida, líquida e gasosa. Uma pequena fração dos componentes da mistura se dissolve na água do lençol freático, uma segunda porção é retida nos espaços porosos do solo na sua forma líquida pura como saturação residual e outra parte dos contaminantes passíveis de evaporação dão origem à contaminação atmosférica (NADIM et al., 1999).

Essa complexidade de contaminação também se deve a uma ampla utilização de diferentes composições de produtos com distintas propriedades, cuja principal característica é a baixa solubilidade e a pouca persistência do solo. Combustíveis como a gasolina e o óleo diesel têm na sua composição os hidrocarbonetos monoaromáticos, como o benzeno, tolueno, etilbenzeno e xilenos, chamados coletivamente de compostos BTEX. Os mesmos são os constituintes que têm maior 
solubilidade em água e, portanto, são os contaminantes com maior potencial de poluir o lençol freático.

Nesse caso, a técnica da biorremediação passa a ser uma alternativa eficiente, pois baseia-se na utilização de micro-organismos para transformar os poluentes em substâncias com pouca ou nenhuma toxicidade.

Para Alexander (1999),

a eficiência desta técnica consiste na capacidade dos degradadores microbianos inoculados ou endógenos permanecerem ativos no ambiente natural. Assim, aumentar a capacidade dos microorganismos degradadores inoculados por meio da bioaumentação ou promover a atividade dos micro-organismos degradadores endógenos por bioestimulação poderia melhorar esta eficiência.

Na bioestimulação, o solo é alterado com nutrientes adicionados, contendo principalmente nitrogênio e fósforo ou fontes de biossurfactantes conhecidos por melhorar a biodisponibilidade dos TPH no local, assim, aumentando a eficiência de biorremediação.

\section{METOdOLOGIA}

O solo contaminado por hidrocarbonetos utilizado na realização do presente trabalho foi coletado durante os trabalhos de recuperação de uma área de um posto de gasolina no município de Porto Alegre. O solo coletado foi acondicionado em uma caixa de PVC, a qual foi dividida em duas partes, de modo que os testes permitissem a comparação entre a eficiência dos processos utilizados. Na primeira parte, identificada como lado $F$, foram adicionadas soluções contendo surfactante CTBA (546 mgl-1, equivalente a $110 \%$ da sua Concentração Miscelar Crítica (CMC), soluções de peróxido de cálcio $(10 \% \mathrm{vol} / \mathrm{vol})$ e o superfosfato $(5 \mathrm{gl}-1)$. Já na segunda parte, identificada como lado $\mathrm{T}$, utilizou-se somente peróxido de cálcio e superfosfato nas concentrações já citadas. A Figura 1 ilustra o sistema montado. 0 desenvolvimento dos micro-organismos nos solos foi monitorado através da contagem das Unidades Formadoras de Colônia (UFC), Bactérias e Fungos, realizada no Laboratório de Diagnóstico Fitossanitário e Consultoria de Porto Alegre/RS. A descontaminação do solo foi acompanhada pela análise dos compostos Benzeno, Tolueno, Etilbenzeno, Xileno, TPH-GRO (Gasoline Range Organics), TPH-ORO (Oil Range Organics) e TPH-DRO (Diesel Range Organics), segundo as normas USEPA 8015D, USEPA 5021A, USEPA 8021B. Para essas análises, amostras de solo foram retiradas em intervalos de no mínimo 30 dias. A duração total dos ensaios foi de 250 dias. 
Figura 1: Divisão do conteúdo da caixa. Fonte: Registro do autor, 2010.

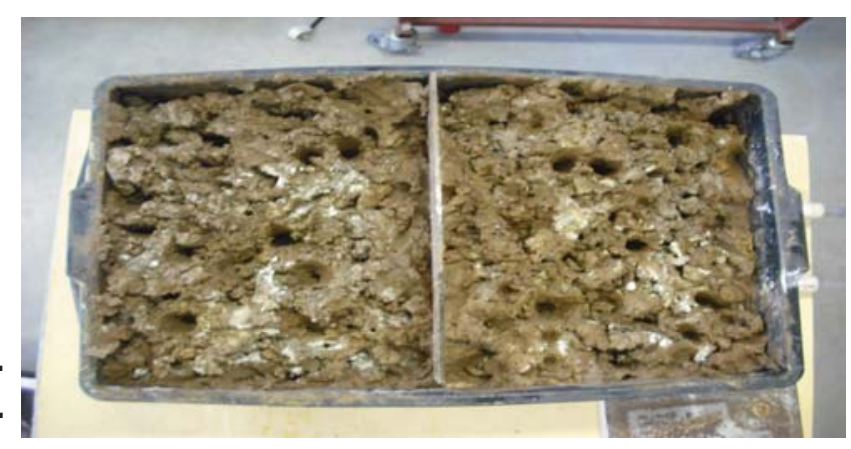

\section{RESULTADOS}

O presente trabalho foi desenvolvido entre agosto de 2009 e julho de 2010, com temperatura média do solo de $17^{\circ} \mathrm{C}$. $\mathrm{O} \mathrm{pH}$ médio foi monitorado nas duas partes do sistema, onde, no lado identificado como $F$, se teve 7,55 durante o período de tratamento de 250 dias. Já o $\mathrm{pH}$ médio encontrado na outra parte, identificada como lado $\mathrm{T}$, foi de 7,8. Durante a realização dos ensaios, observou-se um aumento dos valores de $\mathrm{pH}$, com a aplicação de peróxido de cálcio. $\mathrm{O}$ ajuste e a manutenção do $\mathrm{pH}$ durante o processo de biorremediação é de extrema importância, visto que a maioria dos micro-organismos desenvolvem-se melhor com pH 7,0. Hoffman \& Viedt (1998) reportam que pHs na faixa de 5,5 - 8,5 são ideais para a biodegradação de TPH em solos.

Os resultados da contagem das unidades formadoras de colônia (UFC) de bactérias e fungos, com a presença de surfactante, se encontram detalhados na Tabela 1.

Tabela 1: Resultados da quantidade de bactérias e fungos presente no solo com a presença de surfactante.

\begin{tabular}{ccc}
\hline $\begin{array}{c}\text { Amostra de solo } \\
\text { Contaminado }\end{array}$ & $\begin{array}{c}\mathbf{U F C g}^{-1} \\
\text { de Bactérias }\end{array}$ & $\begin{array}{c}\mathbf{U F C g}^{-1} \\
\text { de Fungos }\end{array}$ \\
\hline Bruto & $1 \times 10^{7}$ & $<5000$ \\
\hline Após 30 dias & $2 \times 10^{8}$ & $1 \times 10^{10}$ \\
\hline Após 60 dias & $1 \times 10^{7}$ & $1 \times 10^{10}$ \\
\hline Após 160 dias & $4 \times 10^{5}$ & Não realizado \\
\hline Após 220 dias & $3 \times 10^{6}$ & $2 \times 10^{3}$ \\
\hline
\end{tabular}

Na Tabela 1, pode-se observar que a população de bactérias apresentou crescimento significativo em relação à amostra bruta no intervalo de tempo de 30 dias, apresentando uma redução com o aumento do tempo de ensaio. $\mathrm{O}$ número final de bactérias após 220 dias de ensaios foi de 3x106 UFC.g-1. Esses resultados demonstram que as condições introduzidas no solo promoveram a bioaumentação das bactérias presentes no solo utilizado, ou seja, a adição de nutrientes e do peróxido como fonte de oxigênio beneficiou a multiplicação dos micro-organismos, os 
quais utilizam o oxigênio dissolvido dos peróxidos sólidos e os nutrientes, assim como os hidrocarbonetos como fonte de carbono, refletindo-se em um aumento da população microbiológica. A redução do número de bactérias presentes no solo pode estar associada a uma redução de substrato disponível para o desenvolvimento dos mesmos, uma vez que a adição de nutrientes e de peróxido continuou nesse período.

Além das bactérias, os fungos desenvolveram um importante papel na degradação dos hidrocarbonetos presentes no solo. Em relação à contagem das UFC dos fungos, realizada na amostra bruta do solo estudado, notou-se que a mesma apresenta um número inicial baixo. Após a aplicação das soluções verificou-se um aumento significativo de fungos presentes no solo ( $<5000$ UFC.g-1). Nos primeiros 90 dias monitorados, observa-se que a concentração de fungos presentes no solo foi a mesma. Quanto à análise realizada ao final do período de monitoramento, observou-se que houve um aumento na quantidade de fungos. Esse aumento pode estar associado ao tipo de substrato presente nesta etapa do trabalho. Após 150 dias de testes grande parte do substrato mais biodegradável (moléculas de baixo peso molecular) já foram consumidas, restando compostos com pesos moleculares maiores, as quais estariam sendo utilizadas pelos fungos, levando a um aumento da população dos mesmos.

Em relação aos compostos BTEX, os resultados estão apresentados na Figura 2, a qual mostra a redução da concentração desses compostos ao longo do tratamento.

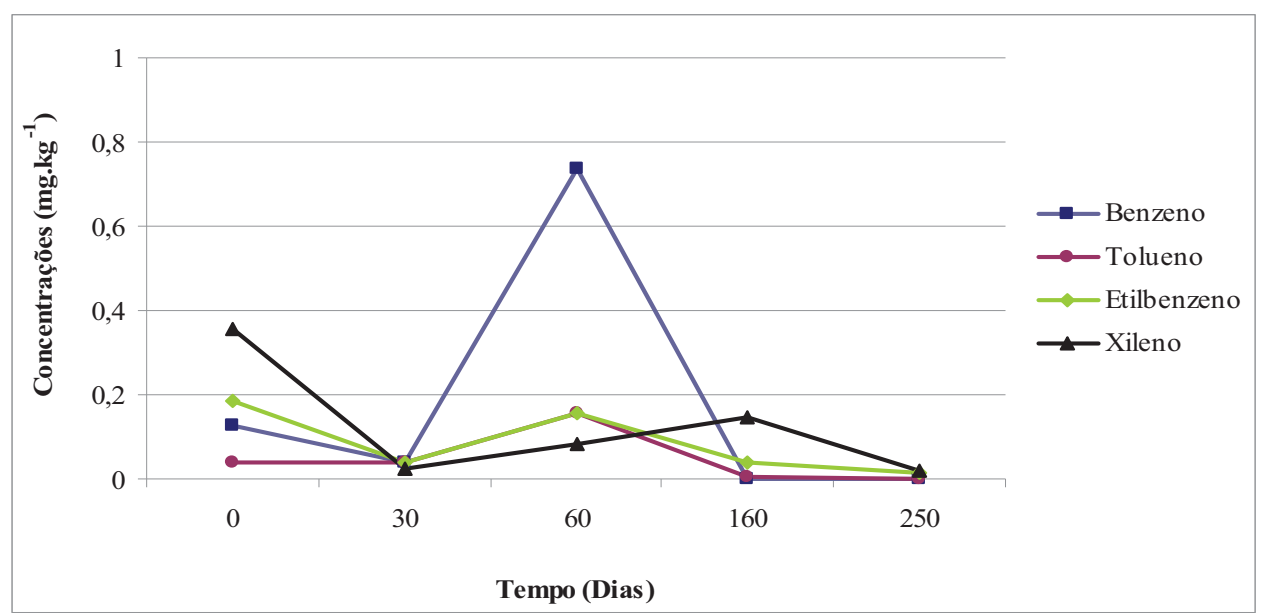

Figura 2: Resultados BTEX do solo contaminado utilizando surfactante

As concentrações encontradas após os 60 dias de monitoramento mostraram um aumento nas concentrações do Benzeno, Tolueno, Etilbenzeno e Xilenos. Esse aumento pode estar relacionado à heterogeneidade do solo.

Ao final do monitoramento observou-se uma grande redução na concentração desses compostos, atingindo uma taxa de degradação de $100 \%, 100 \%, 93,04 \%$ e 94,36\% para o Benzeno, Tolueno, Etilbenzeno e Xilenos, respectivamente. Os valores obtidos atingiram uma concentração abaixo dos valores orientadores da 
Resolução no 420/2009, do CONAMA, que estabelece critérios e valores para a qualidade do solo.

Em relação à degradação dos TPHs, os resultados são expressos na Figura 3.

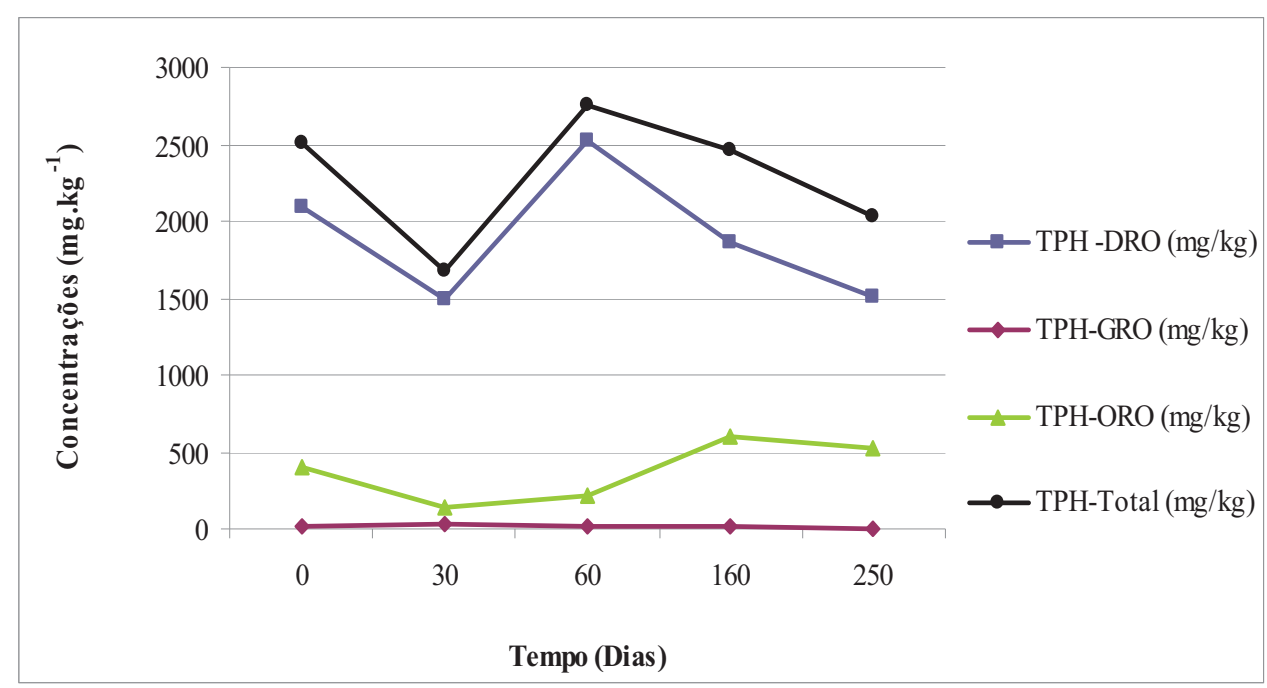

Figura 3: Resultados dos TPHs no solo contaminado utilizando surfactante.

Os resultados apresentados acima mostram uma redução na concentração dos compostos TPH - DRO, ORO e Total. De acordo com os valores obtidos, a remoção do TPH - GRO foi mais eficiente se comparada aos demais compostos, em função de o mesmo apresentar uma maior solubilidade em água.

Em relação aos 60 dias de amostragem, observou-se um aumento nas concentrações dos compostos, o qual também poderia estar associado à heterogeneidade do solo. Ao final do monitoramento, as taxas de degradação dos TPH GRO e DRO foram de $86,16 \%$ e $27 \%$ respectivamente. Da figura, também pode ser observado que o TPH-ORO não apresentou redução. Isso pode estar associado ao tamanho desses compostos, que se constitui de moléculas grandes, dificultando a sua degradação. A partir desses valores, pode-se ressaltar que a degradação do TPH - GRO foi mais rápida se comparada aos demais compostos, uma vez que estaria associada ao baixo peso molecular que o TPH - GRO apresenta, o que o torna mais favorável à biodegradação.

Os resultados da contagem das Unidades Formadoras de Colônias (UFC) de bactérias e fungos para os 220 dias monitorados no solo sem adição de surfactante estão apresentados na Tabela 2. 
Tabela 2: Resultados da quantidade de bactérias e fungos presentes no solo sem a presença de surfactante.

\begin{tabular}{ccc}
$\begin{array}{c}\text { Amostra de solo } \\
\text { Contaminado }\end{array}$ & $\begin{array}{c}\text { UFCg }^{-1} \\
\text { De Bactérias }\end{array}$ & $\begin{array}{c}\text { UFCg }^{-1} \\
\text { de Fungos }\end{array}$ \\
\hline Bruto & $1 \times 10^{7}$ & $<5000$ \\
\hline Após 30 dias & $1 \times 10^{8}$ & $3 \times 10^{10}$ \\
\hline Após 60 dias & $2 \times 10^{7}$ & $1 \times 10^{10}$ \\
\hline Após 160 dias & $1 \times 10^{5}$ & Não realizado \\
\hline Após 220 dias & $2 \times 10^{7}$ & $5 \times 10^{2}$
\end{tabular}

Com relação ao desenvolvimento das bactérias, as análises realizadas no solo demonstraram variações ao longo do período de monitoramento. A população de bactérias apresentou um aumento após 30 dias de testes, após o qual mostra uma pequena redução, porém se mantendo constante até o final do teste. Comparado com os resultados obtidos no solo com adição de surfactante, a população de bactérias desse solo se mostrou mais elevada, o que poderia indicar que o surfactante possui efeitos negativos sobre a população de bactérias. Assim, como já tinha sido descrita anteriormente, a redução no número de micro-organismos pode estar associada a uma redução no substrato disponível no solo, uma vez que a adição de peróxido de cálcio juntamente com o superfosfato continuou nesse período.

Com relação à contagem das UFC dos fungos, verifica-se que após 30 dias de aplicação da solução houve um aumento significativo na população desses microorganismos presentes no solo. Após 160 dias de testes, a população de fungos mostrou uma redução importante, a qual estaria associada à redução dos compostos mais facilmente degradados por esses micro-organismos presentes no solo.

Em relação aos compostos BTEX presentes no solo, os resultados são apresentados na Figura 4.

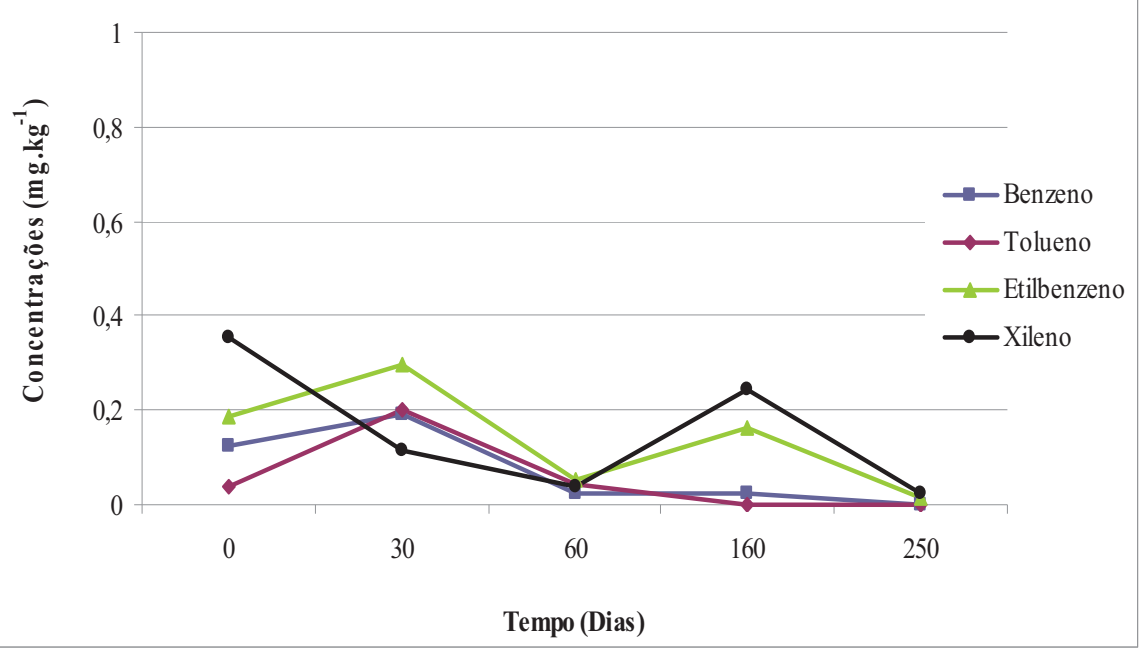

Figura 4: Resultados BTEX do solo contaminado sem surfactante

Revista Jovem Pesquisador, Santa Cruz do Sul, v. 1, p. 49-57, 2010. 
$\mathrm{Na}$ amostra enviada para análise após os 30 dias de tratamento sem a presença de surfactante, foi observado um aumento na concentração de alguns compostos que compõem o BTEX, como o benzeno, tolueno e o etilbenzeno. $O$ xileno foi o único composto que apresentou uma redução nesse intervalo de tempo. Em relação à amostra enviada após os 160 dias de monitoramento, observou-se uma redução significativa na concentração dos compostos BTEX. A taxa de remoção foi de $80,8 \%$ para o benzeno, $100 \%$ para o tolueno, $12,83 \%$ para o etilbenzeno e $30,7 \%$ para os xilenos.

Ao final do período de monitoramento, observou-se reduções importantes desses compostos, ficando as mesmas na ordem de $100 \%, 100 \%$, 93,58\% e 93,52\% para o benzeno, tolueno, etilbenzeno e xileno, respectivamente. Esses compostos apresentaram comportamento semelhante nos ensaios sem uso de surfactante.

Em relação à redução da concentração dos TPHs (DRO - GRO e ORO), os resultados se encontram detalhados na Figura 5.

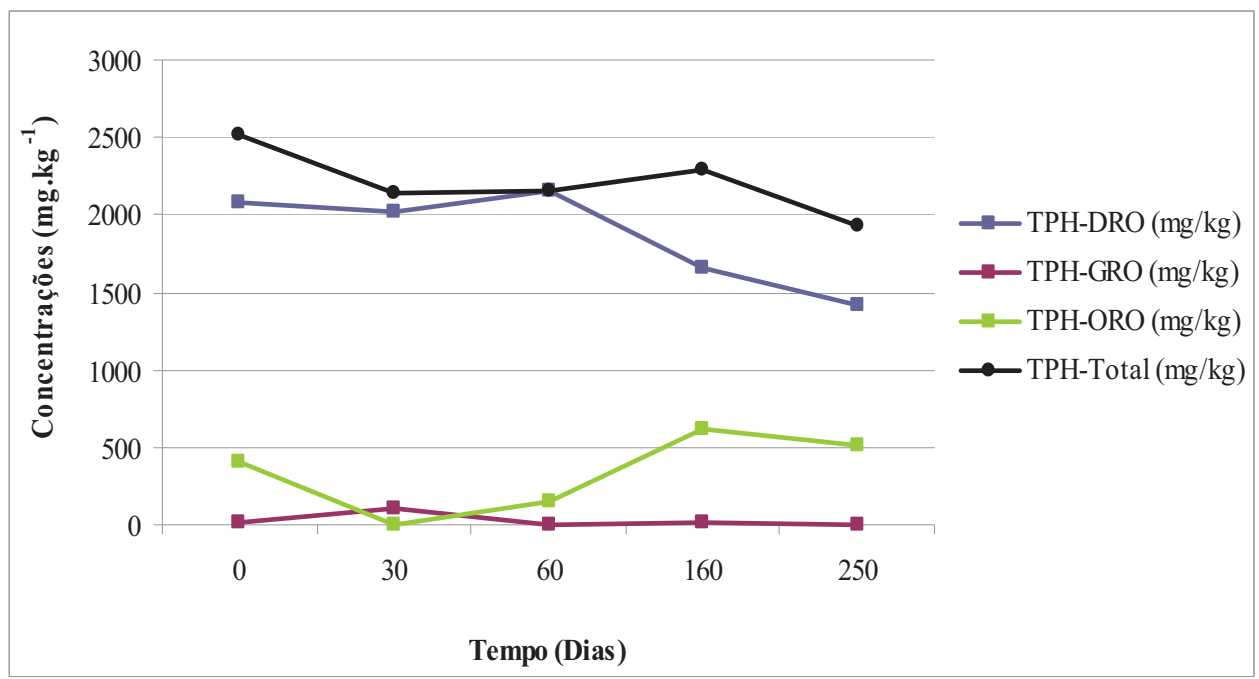

Figura 5: Resultados dos TPHs no solo contaminado sem surfactante.

No intervalo de 0 - 160 dias de testes, não foi observada redução da concentração desses compostos. Após 250 dias de testes, o TPH - GRO apresentou uma taxa de remoção de $91 \%$, o que poderia estar associada à biodegradabilidade desses compostos em função do tamanho de suas moléculas. Nesse período de testes, os compostos do TPH-DRO e Total também mostraram reduções de 32 e $23 \%$, respectivamente. Quanto aos compostos do TPH - DRO e ORO, os mesmos apresentaram uma redução importante de suas concentrações, fato esse de grande importância visto que os mesmos têm por característica possuir grandes cadeias moleculares, dificultando, assim, a degradação desses compostos por parte dos micro-organismos. 


\section{CONCLUSÃO}

A partir dos resultados obtidos ao longo do período de tratamento, observou-se que o lado no qual se tinha adicionado o peróxido de cálcio com o surfactante e nutrientes ocorreram reduções semelhantes dos compostos BTEX e TPHs no solo no qual não se fez o uso de um composto para liberar o contaminante do solo. Nos dois sistemas pesquisados, os valores de BTEX foram reduzidos abaixo dos padrões recomendados pela normativa do CONAMA 420/2009 para solos contaminados.

Em relação aos compostos do TPH, para ambas as partes do sistema analisado, observou-se que, primeiramente, foram consumidos aqueles compostos que apresentaram cadeias menores de carbono (TPH-GRO), e, posteriormente, os compostos TPH-DRO e o TPH-ORO apresentaram uma menor taxa de degradação em função das suas maiores cadeias de carbono.

O oxigênio liberado associado aos nutrientes aplicados no solo gerou, ao longo do trabalho de pesquisa, oscilações na quantidade de micro-organismos, tanto fungos como bactérias.

\section{REFERÊNCIAS}

ALEXANDER, M. In: Biodegradation and Bioremediation. 2nd ed., San Diego, U.S.A., AcademicPress, 1999, p. 453

CONAMA - CONSELHO NACIONAL DO MEIO AMBIENTE, Resolução no 420, de 28 de Dezembro de 2009 - Dispõe sobre critérios e valores orientadores de qualidade do solo quanto à presença de substâncias químicas e estabelece diretrizes para o gerenciamento ambiental de áreas contaminadas por essas substâncias em decorrência de atividades antrópicas.

HOFFMANN, J.; VIEDT, H. Biologische Bodenreinigung Leitfaden für die Praxis. Berlin: Springer, 1998.

NADIM, F.; HOAG, G. E.; LIU, S.; CARLEY, R. J.; ZACK, P. Detection and remediation of soil and aquifer systems contaminated with petroleum products: an overview. J. of Petrol Sci. and Eng., v.26, p.169-178, 1999. 